\title{
«Et on doit prendre parti »: Le film-essai chez Gilles Groulx, en mots et en images
}

\author{
Ignacio Albornoz*
}

\begin{abstract}
Resumo : Tal como a dupla constituída por Pierre Perraut e Claude Jutra, Gilles Groulx representa uma das figuras-chave do cinema contemporâneo do Quebeque. No entanto, o conjunto da sua filmografia continua a ser pouco explorado. O objetivo deste artigo é lançar alguma luz sobre as partes mais obscuras da sua obra a partir do conceito de "filme-ensaio". Mais precisamente, debruçar-nos-emos sobre o filme 24 heures ou plus $(1973,1977)$. Após umas breves discussões conceituais e precisões contextuais, tentaremos mostrar, mediante análise, como se articula em Groulx um pensamento acerca do "filme-ensaio".

Palavras-chave : cinema quebequense ; filme-ensaio; Gilles Groulx.
\end{abstract}

Resumen : Junto al tándem constituido por Pierre Perrault-Claude Jutra, Gilles Groulx representa una de las figuras clave del cine quebequense contemporáneo. Sin embargo, el conjunto de su filmografía sigue siendo poco explorado. El objetivo de este artículo es arrojar alguna luz sobre las zonas más oscuras de su obra a través del concepto de "filme-ensayo". Más concretamente, nos centraremos en el filme 24 heures ou plus (1973, 1977). Tras unas breves definiciones conceptuales y precisiones contextuales, intentaremos demostrar, por medio del análisis, cómo se articula en Groulx un pensamiento sobre el cine-ensayo.

Palabras clave : cine quebequense ; filme-ensayo ; Gilles Groulx.

Abstract : Next to Pierre Perraut and Claude Jutra, Gilles Groulx has established himself as a key figure in the contemporary Quebecois cinema. Despite this prominent position, his filmography is rarely examined in its entirety. The aim of this article is to shed some light on the lesser-known parts of his filmography by attaching it to the concept of "film-essay". More specifically, this article will focus on his film 24 heures ou plus $(1973,1977)$. After a brief definition of concepts and theoretical notions I will explore Groulx's though on the film-essay.

Keywords : quebecois cinema; essay film; Gilles Groulx.

Résumé : Aux côtés du tandem Perraut-Jutra, Gilles Groulx s'est constitué comme une des figures-clés du cinéma québécois contemporain. Toutefois, sa filmographie demeure peu explorée dans son ensemble. Le but de cet article est d'éclaircir ces zones plus sombres du catalogue groulxien par le biais du concept de « film-essai ». Plus concrètement, nous nous pencherons sur son film 24 heures ou plus $(1973,1977)$. Après un bref moment de définitions conceptuelles et de précisions contextuelles, nous tenterons de montrer, par l'analyse, comment s'articule, chez Groulx, une pensée du film-essai.

Mots-clés : cinéma québécois ; film-essai ; Gilles Groulx.

* Doctorant. Université Paris 8 Vincennes-Saint-Denis, Laboratoire ESTCA, Esthetique, Sciences et Technologies du Cinéma et de l'Audiovisuel EA 2302. 93526, Saint-Denis, France. E-mail : ignacio.n.albornoz@gmail.com

Submissão do artigo : 2 juin 2018. Notificação de aceitação : 27 juillet 2018. 


\section{Introduction}

La figure de Gilles Groulx, monteur, réalisateur et scénariste québécois décédé en 1994, demeure aujourd'hui méconnue du grand public, tant en France qu'à l'étranger. Dans les pays hispanophones, elle est presque invisible et se limite, dans le meilleur des cas, à un commentaire supplémentaire accompagnant selon l'occasion les noms de ceux que l'on connaît mieux : Pierre Perrault, Michel Brault ou même Claude Jutra.

À l'instar de l'ensemble du cinéma québécois des années soixante et soixante-dix, l'œuvre de Groulx semble avoir été peu à peu mise à l'écart au profit de nouveaux courants cinématographiques moins directement «engagés » et plus en harmonie avec des thématiques théoriquement plus universelles. En ce qui concerne la France, le sort du cinéma du Canada français est en outre plus que regrettable : ce qui fut, jadis, enthousiasme et fascination pour une pratique bouillonnante dont les possibilités créatives semblaient infinies s'est mué, avec le passage du temps, en une indulgence sereine, non dépourvue de condescendance.

Le présent article se propose de réhabiliter dans la mesure du possible une œuvre du catalogue groulxien par le truchement du concept de « film-essai », duquel ses films seraient - voilà notre hypothèse - des représentants exemplaires. Nous nous intéresserons, plus concrètement, à 24 heures ou plus (1973, 1977), que nous considérons comme un point tournant non seulement de sa filmographie personnelle, mais de l'ensemble du cinéma documentaire québécois de l'époque.

Au long de cette démarche, nous essaierons également de nous appuyer sur les propos du cinéaste lui-même, recueillis notamment dans deux films documentaires d'entretiens qui lui ont été consacrés : Trop c'est assez (1995) de Richard Brouillette et Entretien en six temps avec Gilles Groulx (2002) de Denis Chouinard. L'idée, globalement, sera de montrer comment s'est forgée, tant par la pratique que par la réflexion théorique, une pensée de l'essai chez Groulx. Au long de notre argumentation nous aurons recours, d'autre part, à une série de textes portant sur les caractéristiques de l'essai en cinéma et en littérature. Nous reposerons notre réflexion, principalement, sur des écrits de Jean Starobinski, Marielle Macé et José Moure.

\section{Gilles Groulx, haut personnage à moitié inconnu}

Le silence à l'endroit de Gilles Groulx (ou, stricto sensu, à l'endroit de ses dernières œuvres) est d'autant plus frappant si l'on considère l'exaltation que ses premières incursions dans le cinéma éveillaient chez les critiques de 
l'époque, toutes nationalités confondues. Voici, en guise d'exemple, la brève notice que nous en fournissait, en 1967, le livre Vingt ans de cinéma au Canada français, un petit ouvrage de Robert Daudelin publié avec le concours du Ministère des Affaires Culturelles du Québec. Ses termes sont, pour le moins, flatteurs et ne laissent transparaitre le moindre doute quant à la centralité et l'importance de sa figure :

Le créateur le plus riche et le plus talentueux qui se soit exprimé dans le cinéma québécois est sans nul doute Gilles Groulx (né à Montréal en 1931). Sa sensibilité vive, ses dons de poète, sa conscience même nous ont donné notre premier grand film : Le chat dans le sac. Son œuvre [...] témoigne d'un sens du cinéma peu commun et d'une vision des hommes et du monde qui commande l'attention et le respect. (1967:20).

Malgré l'optimisme de ces premières manifestations de la critique vis-àvis de ses œuvres et la place capitale qu'en apparence lui accordent toutes les histoires du cinéma québécois auxquelles nous avons eu accès, le paysage, sur le plan académique, n'est pas très prometteur. On peine, en effet, à trouver des références bibliographiques à propos du cinéaste et les moteurs de recherche spécialisés ne donnent que quelques résultats, toujours peu satisfaisants car trop axés sur ses films des années cinquante et des débuts des années soixante.

Dans le monumental ouvrage Les cinémas du Canadá : Québec, Ontario, Prairies, côtes Ouest, Atlantique, dirigé par Sylvain Garel et André Pâquet et paru en 1992 chez les Éditions du Centre Pompidou, le nom de Groulx apparaît cité à plusieurs reprises, mais seulement au sujet de ses films de fiction, plus connus : Le chat dans le sac (1964) et Où êtes-vous donc? (1969). Le film auquel nous nous intéressons brille, malheureusement, par son absence. Il en va également de même dans The Cinema of Québec de Janis L. Pallister, par ailleurs très complet. Christian Poirier lui réserve pourtant quelques pages dans son Cinéma Québécois : À la recherche d'une identité ?, sorti en 2004 par les Presses de l'Université du Québec. Son compte-rendu du film, néanmoins, reste superficiel et général.

En tout, un seul ouvrage, paru en 2009 chez Lux, maison d'édition québécoise, fait le point sur 24 heures avec un peu plus de profondeur. Il s'agit de Gilles Groulx, le cinéaste résistant, écrit par Paul Beaucage. Dans ce dernier, on trouve des renseignements précieux sur la vie et les œuvres du cinéaste. Les analyses de ses films majeurs sont fines, aigües et, globalement, très pertinentes. Aussi, l'auteur n'hésite pas à être sévère lorsque la situation le mérite, comme quand il s'agit d'analyser les films les moins réussis de son catalogue. Il s'agit enfin d'un ouvrage très accompli dont le plus grand défaut est peutêtre un certain didactisme et une structure trop linéaire (chaque chapitre est 
voué à l'analyse d'un film) qui pourrait bénéficier d'un rapport plus libre entre les œuvres étudiées.

Deux sources complémentaires présentent, quoique tangentiellement, quelques données à propos de l'œuvre à laquelle nous nous intéressons. Nous nous référons à Le cinéma à l'épreuve de la communauté de Marion Froger, paru en 2009 aux éditions de l'Université de Montréal, et L'Office national du film et le cinéma canadien (1939 - 2003) : Éloge de la frugalité de Caroline Zéau paru pour sa part en 2006 chez Peter Lang, maison d'édition bruxelloise.

\section{La place charnière de Groulx dans le cinéma québécois}

Le manque relatif de visibilité de l'œuvre de Groulx ne saurait exclusivement s'expliquer par un défaut d'intérêt d'un public que l'on pourrait croire peu habitué à des réalisations aussi exigeantes. Ou encore moins - loin s'en faut! - par l'indisponibilité matérielle de ses films, accessibles de manière gratuite sur le site web de l'Office National du Film du Canada (ONF) depuis quelques années.

Le facteur le plus important, croyons-nous, est d'un autre ordre. Il a trait à une suite de décisions controversées et peu heureuses prises au sein de l'ONF et des agences de financement du cinéma québécois, dont les linéaments généraux pourraient se résumer, en des mots de Jeancolas, par l'adage : «moins de films, mais plus d'argent pour chaque film » (1992: 66). Selon Peter Harcourt, en effet, des mesures furent prises par ces institutions à la fin des années soixante consistant à « arracher aux scénaristes et aux réalisateurs le pouvoir décisionnel pour le placer entre les mains des producteurs et des hommes d'affaires [, ce qui] a transformé les films artisanaux à petit budget des années soixante en une industrie du spectacle énorme, mais toujours déficitaire » (1992: 60).

Les effets directs de ces politiques ne furent pas immédiats. Les années soixante-dix, en ce sens, peuvent être considérées comme une étape de transition dans laquelle on observe une marginalisation progressive des entreprises cinématographiques moins traditionnelles ou expérimentales (Harcourt, 1992 : 62). Les questions identitaire et politique, jusqu'à ce moment cruciales, s'effacent alors lentement au profit de courants divertissants et commerciaux importés de l'étranger « dont le seul objectif est de se fondre dans la massification à l'américaine » (Pâquet, 1986 : 114). Dans son diagnostic, Harcourt va encore plus loin et affirme sans gêne que ces politiques ont fini par reléguer le film d'auteur à la « clandestinité » (1992: 62). Yves Lever, pour sa part, partage le même avis, en se servant d'autres mots : « avec les années 70, et surtout 80 , les plus importants budgets sont accordés à la fiction plutôt qu'aux 
essais sociaux, qui ne sont plus réalisés qu'au compte-gouttes » (1995:244). Son constat, pourtant, n'est pas moins révélateur. Pour le chercheur, cette préférence équivaut à bien des égards à une censure tacite, délibérée (Lever 1995 : 244).

En termes plus concrets, on pourrait dire que ces années marquent le moment du passage d'un cinéma si l'on veut artisanal, qui célébrait la précarité en tant que force créatrice, à un cinéma de plus en plus industrialisé et dépendant des diktats esthétiques venant de l'étranger ${ }^{1}$. Qu'ils étaient loin les temps où Groulx pouvait affirmer, avec fierté :

Je me suis toujours dit qu'on pouvait faire un long métrage avec peu d'argent à condition de trouver la manière de le faire, de trouver des ellipses qui ne mettent pas en danger ce que vous voulez, qui permettent d'aller rapidement en se contentant de situations simplifiées au maximum et en refusant toute espèce de scène qui coûterait des jours de tournage ou une équipe trop considérable. (Patenaude, $1964: 6$ ).

Ce tournant s'incarne, concrètement, en une série de crises institutionnelles d'une certaine ampleur dont le point déclencheur fut assurément la conjoncture des événements d'octobre 1970. Le bilan de l'ainsi nommée crise d'octobre, sur le plan social, se clôt par la mort fracassante du ministre Pierre Laporte, la proclamation de la Loi sur les mesures de guerre et l'arrestation «sans motif valable, sinon le délit d'opinion - nationaliste ou socialiste - de près de 460 personnes » (Froger, 2009 : 83). Sur le plan cinématographique, cette crise s'est manifestée de manière également tangible, par la censure ou interdiction, entre 1969 et 1971, de trois films aux partis-pris politiques fort clairs, dont le 24 heures de Groulx, accusé par Sydney Newman, commissaire unilingue de l'ONF, de vouloir mettre en péril la démocratie et le capitalisme canadiens. Pour Yves Lever, cependant, l'explication de l'affaire n'admet aucun doute et demeure transparente : «Seules la peur et la paranoïa héritées d'octobre 70 et conservées dans certains milieux de dirigeants permettent de comprendre cette censure $\gg(1995: 243)$.

Cette vague de censure marque, pour l'ONF, un tournant définitif, au moins en ce qui concerne l'unité de production française (Zéau, $2006: 409$ ), où il devint clair que l'organisme «n'était pas prêt à assumer un rôle dans le débat public, parce qu'il était incapable de donner u statut clair aux cinéastes documentaristes qui œuvraient en son sein » (Froger, $2009: 83$ ). Au niveau individuel, également, ces impasses ont entraîné pour le cinéaste des consé-

1. À ce sujet, Pâquet remarque, non sans ironie : «[...] depuis 1970, notre cinéma a été (à quelques exceptions près) à la remorque de notre propre histoire. SI bien qu'en 1974 nous avons droit à La Gammick [comédie inspirée du film noir américain réalisée à l'ONF par Jacques Godbout] mais non à 24 heures ou plus » (Cité par Zéau, 2006 : 409). 
quences non négligeables qui affectèrent son travail dans le métier, ainsi que sa relation avec les instances de production officielles. Paul Beaucage en fait sommairement le point : " Même s'il réussit à éviter l'emprisonnement [...] Gilles Groulx doit subir les contrecoups de ses prises de positions politiques. Étant soumis à une surveillance incessante, il lui sera de plus en plus difficile de réaliser un film commandité par le service public » $(2009: 171)$.

Les réalisations de Groulx se font alors de plus en plus rares et deviennent davantage audacieuses quant à leur recherche formelle et leur engagement. C'est précisément dans ce contexte de tensions politico-sociales et d'effervescence généralisée que 24 heures voit le jour, se posant certainement comme l'entreprise filmique la plus radicale dans son genre en raison de son effort pour «synthétiser marxisme et nationalisme tout en opérant une révolution formelle »(Lever, 1995 : 382). Le constat, dans le cadre de notre article, n'est pas anodin : les discours essayistes - c'est Irène Langlet qui nous le rappelle dans son très complet L'abeille et la balance - ont tendance à s'épanouir « dans des situations historiques de crise » (2015:24), parmi lesquelles l'auteure compte par ailleurs celle du Québec de l'après-Révolution tranquille ${ }^{2}$.

\section{Le « film-essai », forme du multiple}

Les réflexions autour de l' "essai » au cinéma ont été assez fécondes, bien que peu systématiques. Naturellement, elles se sont nourries de discussions analogues dans le champ de la littérature et de la critique, auxquelles elles ont emprunté une série d'outils théoriques et de concepts de base. A leur instar, néanmoins, les études cinématographiques se heurtent à un exercice dont le risque commun est d'aboutir à des définitions trop génériques qui convertissent parfois le concept en une catégorie à tiroir. La difficulté est d'autant plus prégnante en cinéma qu'il n'y a pas, à la différence de la littérature, de véritable modèle fondateur. José Moure nous le rappelle, d'ailleurs : « [I]l est difficile d'esquisser les contours de cette forme hybride quand, en bonne méthode, aucun élément de définition préalable ne peut être établi, quand aucune œuvre-matrice se dégage » (2004:35).

2. Bien que ses conséquences aient été mises en questionnement par les courants historiographiques les plus récents, la Révolution Tranquille demeure sans doute l'un des événements marquants de l'histoire contemporaine du Québec. On entend sous ce terme, globalement, la série de processus sociohistoriques de modernisation et sécularisation qui ont façonné la société québécoise de la seconde moitié du siècle. Plus concrètement, la Révolution Tranquille désigne tout un éventail de réformes mises en œuvre à partir de 1960, année de l'élection de Jean Lesage comme Premier ministre du Québec. Ces réformes se sont traduites par une plus grande intervention de l'État dans les affaires nationales et ont touché, en somme, à des aspects tels que « [la] démocratisation politique, [la] modernisation et laïcisation des institutions, [la] justice sociale, [la] liberté d'expression, [1'] accès à l'éducation et aux soins hospitaliers, [le] soutien à la culture, [et la] fin de la censure » (Comeau, 1995 : 52). 
Sans vouloir trancher ici la question de manière définitive, nous nous bornerons tout simplement à relever quelques caractéristiques ou attributs essentiels que nous avons pu collecter dans les nombreux articles et ouvrages qui s'y adonnent. Ce afin de les mettre à l'œuvre, par l'analyse, dans le film qui nous occupe. Nous allons commencer, d'abord, par certaines considérations d'ordre général ou théorique, que nous ponctuerons à l'aide de quelques remarques du réalisateur.

Alexandre Astruc, écrivain et cinéaste français, a été peut-être l'un des premiers à mettre en équivalence des productions cinématographiques contemporaines avec la notion, jusqu' alors exclusivement littéraire, d'essai. Dans son article de 1948, L'Avenir du cinéma, il constatait ainsi l'émergence d'un cinéma qui se défaisait du fardeau du spectaculaire et acquérait un statut nouveau, celui d'un art « qui pourra s'adresser davantage à l'intelligence» $(1992: 331)$ : «Le cinéma qui est en train de naitre se rapprochera beaucoup plus du livre que du spectacle, sa langue sera celle de l'essai, poétique, dramatique, dialectique, tout à la fois » (ibid : 331). Le recours à cette notion issue du champ des lettres servait à Astruc, surtout, pour poser la question, à son avis très prégnante, sur la « maturité » du cinéma; c'est-à-dire, sur sa capacité d'exprimer, au même titre que la peinture ou le roman, «n'importe quelle pensée humaine » (ibid : 328), sur sa capacité de devenir, enfin, « forme d'expression » (ibid : 330) à part entière.

Prenant appui sur les conceptions d'Astruc, Christa Blümlinger nous rappelle pour sa part que le film-essai, en tant que « flânerie intellectuelle », possède un caractère « provocateur, voire parfois destructeur» $(2004: 53)$. Ses armes, en ce sens, « [...] sont l'humour, l'ironie et le paradoxe; son principe est la contradiction, la collision » (ibid : 54). Citant en substance Adorno, pour qui l'essai refléterait « le loisir propre à l'enfance » (2003:51), Blümlinger souligne, aussi, l'aspect ludique du genre : « [...] la chance et le jeu lui sont essentiels, il s'abandonne à un loisir enfantin et s'enflamme sans scrupule à ce que d'autres ont déjà fait » (2004: 54).

Il est intéressant de constater, ici, la similitude que la métaphore utilisée par Adorno entretient avec les termes employés par Groulx lui-même pour expliquer sa méthode de travail en salle de montage. Groulx se sert, en effet, de l'image du jeu, qu'il développe à sa manière. Ainsi, lorsqu'il remémore, dans Trop c'est assez, ses temps à l'ONF, il dit : "On était des enfants. Ce qu'il faut être. On était désobéissants et malvenus. Enfin, on était nouveaux, on n'était pas conformistes, ni catholiques [...], on allait à tâtons ».

Il conviendrait de mentionner, d'autre part, une certaine discontinuité, une fragmentation délibérée du discours qui déterminent cette nature de pensée 
en train de se faire (Blümlinger 2004 : 54), très caractéristique du genre de l'essai. Ce caractère de chose non-résolue est bien résumé par Groulx lorsqu'il s'étend, au cours d'une émission télévisuelle, sur les relations troubles entre engagement politique et documentaire. «Le manifeste », dit le réalisateur à ce propos, «c'est un problème avec sa solution ». Puis il précise : « Mais le film n'apporte pas de solutions. Le film pose la question. Dans le cinéma comme je le conçois, c'est le film qui pose la question $»^{3}$ (Chouinard 2002).

Dans un autre ordre d'idées, le concept de film-essai désigne le statut particulier d'une œuvre donnée qui l'éloigne, par contraste, des productions environnantes. En ce sens, Suzanne Liandrat-Guiges a bien raison lorsqu'elle affirme que « la qualité d'essai devient l'unique possibilité de désigner le cinéma qui résiste face aux productions commerciales » $(2004: 10)^{4}$. Le cinéma de Gilles Groulx semble répondre assez aisément à cette dernière distinction. C'est d'ailleurs le réalisateur lui-même qui le souligne, lorsqu'il se réfère à l'état général du cinéma de son époque, envers lequel il est d'ailleurs fort critique. Ses Propos sur la scénarisation, bref ouvrage rédigé dans le cadre d'un cours de création cinématographique du Collège Montmorency réalisé en 1976, fournissent une preuve éloquente de la place qu'il assigne au cinéma qui l'intéresse, et qu'il conçoit comme étant aux antipodes des courants dominants de l'industrie : « [I]l est tout de même évident que les films qui rendent une vision claire des choses que nous vivons se font très rares ; ils deviennent, pour ainsi dire, marginaux $»(1986: 15)^{5}$.

3. Cette idée du cinéma en tant qu'exercice rhétorique de formulation d'une question à répondre apparaît par ailleurs chez Groulx de manière fort transparente. Rappelons à cet égard qu'au moins deux de ses films portent, dans leur titre, un signe d'interrogation (Où êtes-vous donc?, Québec?) et que deux autre (24 heures ou plus, Entre tu et vous) proposent de façon assez nette une sorte de dichotomie à résoudre, une disjonction péremptoire.

4. Une autre pionnière de la spéculation théorique autour du cinéma, Germaine Dulac, allait aussi dans cette direction. À lire ses Écrits sur le cinéma on perçoit que l'auteure s'efforce de donner une image complexe du cinéma, qu'elle conçoit comme étant à mi-chemin entre l'industrie et l'art. Or, malgré ce parti pris, des catégorisations divergentes émergent. Le champ cinématographique se diviserait ainsi, pour Dulac, en un versant récréatif, aux ambitions populaires, et un versant « d'essai » (ou « d'évolution »), dont la tâche serait de pousser toujours plus loin les limites du visible et duquel déprendrait, en dernière analyse, le cheminement vers l'avant de l'art filmique. "Ceux qui s'en occupent », affirme Dulac, « sont dédaignés par les grands industriels du film qui n'admettent pas les expériences à côté de la production normale » (1994:167).

5. Pour ce qui est de son mécontentement vis-à-vis de l'aire cinématographique de son temps, Groulx a été, en effet, assez franc. Ainsi, et toujours dans ses Propos, il affirme : «Aujourd'hui, en 1975, je crois que dans le commerce que l'on fait du cinéma [...] nous sommes revenus au nécessaire conformisme des années 50 ; des scénarios ramenés au linéaire du récit conventionnel, pour des films sans originalité et sans conviction, qui piétinent dans l'hébétude » (1986 : 14). À la lumière de ses déclarations, il s'avère clair que le cinéma de Groulx est animé d'un désir d'aller à l'encontre des pratiques issues de ce qu'il appelle l' « idéologie régnante », laquelle cherche, selon ses propres mots, à « ériger le conservatisme en tradition, [et à] contrôler le cinéma » (ibid : 2), par le biais d'une approche trop axée sur les profits et le divertissement. 
Le cinéaste n'hésite pas à emprunter des termes plus percutants lorsqu'il s'agit d'évoquer l'opposition entre le type de cinéma qu'il prône et celui qui semble dominer l'industrie, entre lesquels il constate par ailleurs l'existence d'un gouffre idéologique indépassable :

Il est bien évident qu'ériger la notion capitaliste de profit en tant qu'art est incompatible avec l'expression libre, la volonté d'innover et de discuter les idées. Ce n'est pas d'une lutte entre un cinéma plus facile ou plus difficile qu'il s'agit, mais de deux idéologies opposées : entre conformisme et découverte ; entre dirigisme et liberté. (Groulx, $1986: 5$ ).

Prenant appui sur l'expérience de Dziga Vertov, qu'il citait fréquemment, le cinéaste québécois reconnaît, tant dans sa pratique que dans ses nombreuses interventions écrites, qu'un cinéma conçu comme « [...] instrument d'information/éducation » est voué en quelque sorte au mépris ou au discrédit : «En 1975, un cinéma issu de cette conception subit les mêmes avatars [qu'à l'époque de Vertov] : marginalité, condition de pauvreté des productions, manque de continuité, etc. ${ }^{6}$ (Groulx, $1986: 2$ ). Pourtant, cette position de marginalité, résultat du manque de visibilité des moyens d'expression choisis, Groulx la revendique, avec fierté, comme la seule condition possible d'un cinéma affrontant la réalité en tant que telle.

Mais revenons aux caractéristiques du film-essai, que nous avons quelque peu oubliées. Nous voudrions retenir, pour la définition de ce genre, d'autres traits essentiels qui nous permettront de mieux saisir ses enjeux. Dans son livre Le cinéma, autrement, Dominique Noguez, théoricien du cinéma, en soulève quatre. Nous les consignons ici, sommairement, en raison de leur caractère synthétique $^{7}:$ «1) un parti-pris de non-narrativité et [...] un recours abondant à la citation sous toutes ses formes (images, sons, textes écrits) ; 2) un caractère subjectif (donc incertain, ouvert) affiché ; 3 ) le souci exclusif de la réalité présente ou à venir ; 4) la volonté d'infléchir par des moyens rationnels l'opinion du spectateurs, de l'amener à une prise de conscience, voire de changer sa vie $\gg(1987: 293)$.

On pourrait encore adjoindre, aux quatre traits que nous venons d'évoquer, quatre autres, recueillis dans la littérature. Nous en faisons la liste promp-

6. C'est par ailleurs toute la condition du cinéma québécois dans son ensemble - « cinéma dominé s'il en est un », dira Groulx lui-même - qu'est désignée par ce dernier constat. Groulx est en effet fort critique vis-à-vis des possibilités réelles d'autonomie du cinéma fait au Québec «Un cinéma national québécois qui ferait foi des questionnements et des luttes de ceux qui veulent s'affranchir de la domination et de l'autoritarisme ne peut guère à mon avis trouver preneur dans nos gouvernements actuels » (1986:15).

7. Ce qui surprend est, d'autre part, que Noguez ouvre justement sa définition du film-essai par une référence à Gilles Groulx, auquel elle reviendra à plusieurs reprises dans le même chapitre. Plus spécifiquement, l'auteure se réfère au film Entre tu et vous, qu'elle classe, aux côtés du Gai Savoir de Godard, comme un exemple privilégie des possibilités esthético-rhétoriques du genre. 
tement, sans trop s'attarder sur les détails, prenant appui sur des textes de Liandras-Guigues, José Moure et Marielle Macé. Dans le film-essai il existerait : 5) un brouillage, incarné par l'apparition non fortuite du réalisateur à l'écran, du « [...] partage entre texte et paratexte » (Liandras-Guigues 2004 : $10)$, ou, si l'on veut, entre narration et commentaire; 6) un recours au dialogisme, servant à suspendre la narration traditionnelle; 7) un certain goût pour le « mémorable », par l' « exemplaire », par le « citable »; en somme, par la force de frappe d'images-pensées qui décident « [...] de l'inscription de l'essai dans une temporalité culturelle qui l'englobe et le dépasse » (Macé 2008 : 7).

\section{4 heures ou plus : l'essai à l'œuvre}

Une partie non négligeable de la bibliographie portant sur 24 heures ou plus repose sur l'étude d'un fait quelque peu accessoire : sa censure et l'interruption de sa production par l'alors commissaire de l'ONF Sydney Newman, qui retarda de presque cinq ans sa sortie. Sans vouloir ignorer les conséquences et les préjudices que cette impasse a entraîné tant pour le réalisateur lui-même que pour la réputation de l'équipe de l'ONF, nous tenons cependant à dédramatiser son importance pour l'interprétation du film. En effet, il faut bien noter que ce cas est loin d'avoir été le seul dans son genre, la censure ayant touché, dans sa forme la plus ouverte et directe, d'autres cinéastes de renommé, comme Denys Arcand et Jacques Leduc ${ }^{8}$. Force est de constater, comme nous le rappelle en outre Yves Lever, que la censure a été une pratique courante au sein de l'ONF. La seule différence réside dans le fait que les voies qu'elle empruntait avaient au moins, auparavant, la vertu d'être plus subtiles (1995 : 243).

En ce sens, nous voudrions revenir à une question si l'on veut plus indispensable et concrète. À savoir : quels sont les attributs qui, dans l'œuvre de Groulx, et plus spécifiquement dans 24 heures ou plus, autorisent l'appellatif de « film-essai »? En guise de réponse, nous proposons au lecteur de procéder par découpage, en isolant et reprenant, dans le même ordre dans lequel elles ont été évoquées, les caractéristiques que nous venons de consigner plus haut. Nous illustrerons chacune de celles-ci à l'aide de séquences précises.

(1) Non-narrativité et recours à la citation : Le film-essai, nous dit José Moure à propos des films télévisés de Rossellini, se caractérise par le « recyclage de matériaux différents » (2004 : 30). Le constat, on le voit, s'adapte assez confortablement à 24 heures ou plus. Ce qui y fait saillie au premier re-

8. Pour une vision plus approfondie du phénomène de la censure à l'ONF, nous renvoyons le lecteur à l'ouvrage précité L'Office national du film de Caroline Zéau. Elle y traite plus amplement de ces questions, dans un des chapitres finaux du livre, consacré aux films interdits. 
gard est en effet la multiplicité de sources visuelles et sonores mises au service du message transmis : images mouvantes, images fixes, textes écrits, voix-off, enregistrements sonores pris sur le vif, etc. On remarque également la diversité de genres discursifs ou de situations énonciatives que ces mêmes sources ont pour but d'illustrer : journaux, entretiens, discours politiques, communiqués de presse et adresses directes aux spectateurs. Enfin, toute une série d'images hétéroclites est mobilisée dans le film pour atteindre à un seul et même but : la dispersion, le recoupement, le déplacement et la mise en «errance » des repères temporels d'un récit qui - c'est le titre lui-même qui nous l'annonce - était censé nous donner à la base un aperçu d'un intervalle de temps fort précis : les mois de novembre et décembre 1971.

Les exemples de ces matériaux hétéroclites font légion et ils tiennent tant à la texture qu'au chromatisme d'une image qui se veut plurielle, diverse. En effet, le film entremêle - sans qu'on puisse pour autant en déceler les raisons - des séquences en noir et blanc avec des séquences en couleur. Ces dernières, moins fréquentes que les premières, ont toutefois, en raison leur rareté, une plus grande force, bien que leur emplacement demeure arbitraire. Une de ces séquences en couleur nous montre par exemple le témoignage, en plan très serré, d'un employé « [...] dont la santé a été altérée par le fait qu'il travaillait dans des conditions professionnelles inacceptables et périlleuses » (Beaucage, 2009 : 190). Le travailleur dit tout son découragement avec des mots simples, qui traduisent avec probité et transparence un vécu « [...] dont on ne saurait en aucun cas remettre en question la véracité » (ibid : 191).

Un peu plus tard, l'objectif de la caméra se pose sur l'écran d'un appareil de télévision, dont les bords ne sont pas dissimulés. À l'image, on voit l'alors Premier ministre du Québec Robert Bourassa, qui s'étend - la critique de Groulx, ici, est brûlante - sur l'une de ses activités sportives favorites : la natation. Le plan, cette fois-ci en noir et blanc, nous apparaît davantage impersonnel, car médié par un dispositif second, interposé : celui de la télévision. Le même procédé est utilisé d'ailleurs pour montrer une conférence de presse de Pierre Elliott Trudeau, pendant laquelle celui-ci si s'adresse aux citoyens au sujet des relations bilatérales entre le Canada et les États-Unis. Le contraste, on le voit bien, cherche à illustrer, par l'image, la présence d'un gouffre entre deux régimes de paroles, par rapport auxquelles le réalisateur n'hésite pas à se situer : là où le témoignage de l'ouvrier (en couleur) revêt donc un aspect familier, spontané, presque d'une certaine douceur, les allocutions des deux hommes publics sembleront, en revanche, verrouillées et artificielles, affectées. 
On pourrait parler également des nombreuses coupures de journaux qui rythment et ponctuent le film. Elles se multiplient tout au long du récit, en le densifiant, en y ajoutant des couches de sens. Leur apparition, pourtant, n'obéit pas à un but purement illustratif. Pour la plupart, elles viennent interrompre inopinément le déroulement d'une séquence, sans chercher à renforcer quelque idée que ce soit. Le montage, en d'autres cas, est tellement accéléré qu'on n'a même pas le temps de lire ou d'identifier les titres des articles. Immédiatement après le discours de Pierre Elliott Trudeau que nous venons d'évoquer, par exemple, Groulx dispose, l'une après l'autre, plus d'une vingtaine de coupures de journal et photographies de sources différentes. Les premières d'entre elles renvoient, ironiquement, et dans un but de dénonciation, à la fausseté des propos tenus par Trudeau. Elles sont là pour nier tout ce que le Premier ministre affirmait avec tant de conviction dans le plan précédant.

Commence ensuite une dérive qui amènera à une dispersion des thèmes traités. Peu à peu, on verra donc défiler des unes traitant de sujets aussi divers que l'accueil de Fidel Castro au Chili par le président Allende ou les discours des mandataires chinois à l'ONU, l'ensemble étant ponctué par des photographies dont les sources ne nous sont pas non plus révélées. Comble de la libre association, la séquence se termine par l'image en couleur d'un piège à souris se fermant violemment sur un drapeau américain. Vient ensuite, pour tout clore, un rapport qui détaille les dépenses en publicité des principales entreprises américaines, sur lequel la caméra s'arrête pendant une bonne dizaine de secondes.

(2) Caractère incertain, ouvert, subjectif : L'essai cinématographique, affirme Murielle Gagnebin, obéit à « [...] un plaisir spécifique : celui de l'essayage, du libre mouvement qui anime toute structure ouverte » (2004:17). Cet aspect d'incertitude, d'ouverture, curieusement, c'est aussi le titre qui nous l'annonce, au moyen d'une dichotomie non résolue. La formule « 24 heures ou plus » est tirée d'un discours du leader syndical Michel Chartrand, prononcé à l'occasion d'un rassemblement syndical ayant eu lieu le 2 novembre 1971. Dans ledit discours, Chartrand, charismatique qu'il était, exhorte le public par le récit des exploits du système capitaliste, le paroxysme de l'allocution étant le moment - coupé dans la version finale - où il invitait les auditeurs à une grève générale qui pourrait, dit-il, durer « 24 heures ou plus » (Zéau, 2006 : 406). Promesse, menace ou augure, la formule sert aussi à suggérer la possibilité d'une prolongation de la grève - et par là même du film - au-delà de ses limites prévues originalement.

Il n'est d'autre part guère étonnant que le film s'ouvre par une séquence condensant à sa manière l'idée d'un travail en cours, d'un mouvement perpé- 
tuel, ce qui vient accentuer l'idée, déjà évoquée, du film-essai comme pensée non-close. Le tout premier plan nous montre en effet, à l'aide d'un plan fixe, un paysan-ouvrier, situé à quelques mètres de distance de la caméra, qui est d'ailleurs fixe sur son axe. Le paysan remue de la terre à l'aide d'une fourche. L'opération est plutôt silencieuse, sobre. Au loin, quelques objets attirent l'attention, bien que la durée du plan ne permette pas de les reconnaitre tous : à droite, une vieille maison de campagne en bois; au centre, un grand chariot de transport; à gauche, au fond de l'image, une sorte de grenier. La bande-son, quant à elle, est fort discrète. Il n'y a que le bruit du vent qui fait saillie, coupé par moments par les bruits et klaxons des voitures qui passent sur une route que l'on peut supposer proche. La scène renvoie certainement à un temps vide, immémorial - celui du travail manuel, pré-mécanique, que l'on pourrait croire révolu. Une légende en sous-titre nous indique pourtant qu'il s'agit bel et bien d'une scène contemporaine, tourné le $1^{\text {er }}$ novembre 1973.

Il est encore moins étonnant que ce soit justement à l'intérieur de cette séquence que l'objectif et le dispositif du film nous soient révélés, comme s'il s'agissait pour Groulx de mettre en équivalence le labeur manuel du paysanouvrier et celui du cinéaste, à mi-chemin entre le geste manuel et la technique. Sitôt le plan d'ouverture fini, on entend, sur la bande son, le bruit constant, percussif, d'un train qui parcourt à vitesse moyenne (il vient assurément tout juste de quitter la gare) une voie ferrée. La caméra, située à l'intérieur du wagon du train, montre à son tour un paysage banlieusard, gris, à l'allure industrielle. Par ce geste fort métaphorique de « mise en mouvement» du récit - que Beaucage lit, à son tour, comme un singulier clin d'œil au genre du road-movie - Groulx donne le signal de départ à un film qui, bien que ne comprenant qu'une parcelle réduite de temps, possède déjà en soi la possibilité d'une continuation, car « [...] il se construit tout en se révélant» (Terrier Hermann, 2010 : 94). Inutile de rappeler que la toute première intervention de Groulx se clôt elle aussi sur une note d'ouverture : « Ce film est en suspens, car son dénouement dépend de nous tous ».

(3) Souci du présent, projection vers le futur : «Suis-je allé à la rencontre du monde présent comme l'a fait Montaigne en son temps? », se demandait Jean Starobinski (2003 : 179) lorsque le Prix Européen de l'Essai Charles Veillon lui était décerné en 1982. Toute proportion gardée, cette même question, nous parait-il, guide l'entreprise de Gilles Groulx dans 24 heures ou plus : comment aller, par le biais de l'image et du son, à la rencontre de son temps?

Il convient de rappeler d'ailleurs, comme le fait Murielle Gagnebin, que le mot « essai », de par la racine étymologique ex-agere, désigne le fait de 
« conduire au-dehors », « vers quelque ailleurs l'esprit, le regard ou la psyché » (2004 : 15). En peu de mots, on peut dire que l'essai a une vocation de projection, que ce soit dans le temps ou dans l'espace. Vocation qui répond, selon les mots de Starobinski, à une « curiosité infinie pour le monde extérieur » (2003 : 175), dont Montaigne, maitre et forgeur du genre, était dépositaire.

Dans le cas de 24 heures, la problématique de la projection vers le futur et le souci du temps présent sera posée et figurée avec détermination. La séquence qui clôt le film en est fort exemplaire. Si le récit - nous venons de l'évoquer s'ouvrait par la lente mise en mouvement d'un train qui quittait une gare sous fond d'un paysage industriel, la séquence finale - intitulée « retour à Montréal $»$ - en fera autant, sauf que cette fois-ci la caméra sera placée à l'intérieur d'une voiture qui traverse le pont Jacques Cartier. Et l'image, à la différence du plan d'ouverture, sera en noir et blanc.

Il convient de noter encore une autre différence : si la séquence d'ouverture capturait le paysage de côté - les fenêtres du train ne pouvant pas offrir, par leur disposition, une vue du chemin à parcourir -, celle de la fin nous fournira, en revanche, une vue de front, s'étendant vers l'avenir. L'ensemble sera rythmé, de surcroît, par le mouvement parallèle des essuie-glaces du parebrise, qui ne cessent pas de bouger. Ceux-ci nous rappellent - la métaphore du passage du temps est ici fort transparente - toute une série de stimuli sonores et visuels vus ou entendus au long du film et renvoyant à une certaine idée d'automatisme, de mécanicité : les aiguilles d'une horloge, le roulement des roues ferroviaires, le martèlement d'une perceuse industrielle, etc. Par le biais de ce geste d'opposition symétrique, le récit se ferme donc dans une boucle parfaite par laquelle le réalisateur indique, selon Beaucage, « la fin de son itinéraire cinématographique, de son roadmovie métaphorique » (2009 : 201).

La métaphore de la route, accomplie concrètement par le mouvement du train et de la voiture, s'avère en outre très parlante si l'on considère son emplacement dans les deux «bords » du récit : le début et la fin. Son interprétation, d'ailleurs, nous semble en cela assez transparente : d'une part, le film, dans son mouvement, regarde le présent, qu'il capture, étudie et commente en temps réel (voilà pourquoi le récit commence dans le train, avec une caméra qui filme perpendiculairement le trajet du véhicule); d'autre part cette référence à la scène du début, 24 heures se projette vers un futur possible, auquel la caméra fait face de front, à travers le pare-brise, avec, malgré les apparences, « un optimisme dépourvu de naïveté » (Beaucage, 2009: 201). Le geste, d'une simplicité foncière mais éloquente, clôt le récit «sur une note d'ouverture » (ibid : 201), transformant cette fin apparente en un nouveau commencement. Et Starobinski de renchérir, à propos des caractéristiques de l'essai : « cette allure 
de commencement, cet aspect inchoatif [...], sont assurément capitaux, puisqu'ils impliquent l'abondance d'une énergie joyeuse qui ne s'épuise jamais en son jeu» $(2003: 170)$.

(4) Apport à une prise de conscience du spectateur : Cela va un peu de soi, mais le film-essai - Véronique Terrier Hermann, parmi tant d'autres, nous le confirme - n'est pas indifférent à la contingence. Au contraire, il y participe activement : «N'ayant pas vocation à rester neutre, le film-essai [...] peut être partisan, polémique et s'affirme comme prise de parole assumée » (2010 : 92). Irène Langlet, pour sa part, nous rappelle que l'essai, en tant que genre littéraire, peut dans certains cas être apparenté au pamphlet, ou bien à l'écrit, au discours polémique (2015: 59).

Quoi qu'il en soit, il apparaît évident que, dans 24 heures ou plus, Groulx et le politologue Jean-Marc Piotte « [...] énoncent de façon univoque leur point de vue » (Beaucage, 2009 : 186). Or, ce qui importe de souligner est plutôt comment Groulx réussit à renforcer son point de vue et la prise de conscience du spectateur par un agencement astucieux, catégorique et soigneusement conçu de ses adresses verbales directes, qu'il dispose à des points précis du film, et non exclusivement par leur contenu. Examinons, en guise d'exemple, la fin du film, où Groulx choisit de suivre une logique récapitulative s'appuyant sur le sens commun et sur l'observation de la réalité. Il affirme, alors : « Nous croyons qu'il faut remettre en question le système établi. Notre vie, nos observations, ces images que nous avons filmées, tout nous convainc que les structures actuelles sont incompatibles avec une société où les travailleurs contrôleraient leur travail, leur production et où le développement économique serait orienté en fonction des besoins de l'homme ». Ce bilan final, qui fait très nettement le point des témoignages exposés tout au long du film, n'a autre but que celui d'inciter le spectateur à mesurer « dans sa propre vie quotidienne, en quoi il peut connaître des expériences comparables à celles des gens que l'on voit apparaître dans 24 heures ou plus » (ibid : 201).

Or, par le truchement de ce procédé argumentatif, Groulx nous suggère, non sans malice, que les images qu'il a agencées, tout en restant ouvertes à l'interprétation, conduisent par elles-mêmes, au moins dans un premier temps, à une seule et unique lecture contingente. Mais il a - et c'est en cela que réside la vertu du film - une singulière manière de le suggérer. Car, en plaçant ce commentaire à la toute fin, la conclusion du cinéaste se présente comme celle que tout un chacun aurait pu tirer devant un tel spectacle de réalité. Barbara Ulrich, sa conjointe, nous le confirme d'ailleurs dans Voir Gilles Groulx, documentaire de Denis Chouinard. La comédienne nous rappelle pertinemment que lorsque la direction de l'ONF a demandé au cinéaste de changer le monologue 
de la fin du film, il a refusé, en arguant : « Ce n'est pas moi qui dis ces choseslà, c'est toutes les personnes qui jouent dans le film. Je ne peux pas faire une analyse autre que celle qui est révélée par tous les gens » (2002).

(5) Brouillage de texte et commentaire : Art de l'équilibre par antonomase, le film-essai serait, selon Suzanne Liandrat-Guigues, un jeu d'oscillations, « un laboratoire où se trouvent miniaturisés l'univers du cinéaste ainsi qu'un certain monde filmique » (2004: 12). Mais équilibre entre quoi, au juste? Tout d'abord, entre les forces gravitationnelles de ces deux extrémités auxquelles on a tendance d'attacher toute œuvre filmique : la fiction et le documentaire. Dans l'œuvre de Groulx, on le sait, ces deux courants interagissent dynamiquement, brisant toute convention générique depuis ses premiers films. Dans 24 heures ou plus, leur tensions sont davantage mises en crise, ce qui amène Paul Beaucage à affirmer, avec raison, que 24 heures « est un documentaire qui contient une indéniable part de fiction, [...] [laquelle] se rattache fréquemment à l'imaginaire des intervenants » (Beaucage, 2009 : 183). Néanmoins, nous assure-t-il - et nous acquiesçons -, « cet imaginaire ne prend jamais le pas sur l'essence de la réalité » (ibid).

Comment cet équilibre précaire entre l'imaginaire des intervenants et la « présence obtuse » du réel se maintient-il alors dans 24 heures ? Par un tout particulier procédé de brouillage entre le texte (compris ici au sens large du terme) et les commentaires émis par le cinéaste et son collègue Jean-Marc Piotte. Ce brouillage, nous paraît-il, est très ingénieusement mené à terme par Groulx au moyen d'un procédé d'incrustation intermittent, qui lui sert, de temps à autre - et assez inopinément - à introduire des remarques critiques, des mots d'esprit de nature diverse. Le dispositif d'incrustation, on tient à le souligner, avait déjà été annoncé au tout début du film, lorsque le réalisateur nous révélait lui-même, en s'adressant à la caméra, la méthodologie de tournage suivie : «Dans un esprit, de solidarité ou de dénonciation, disait-il alors, nous avons rédigé un certain nombre de courts textes qui, dans le déroulement du film, peuvent apporter quelques éclaircissements ».

Ici, encore une fois, la métaphore choisie par Groulx pour expliquer son procédé n'est pas sans rappeler certaines des caractéristiques que Jean Starobinski reconnaissait chez Montaigne - notamment son humilité stratégique, son refus de toute «[...] matière à litige doctrinal » (2003 : 169). En effet, à l'instar de l'auteur des Essais, Groulx n'estime pas que ses commentaires puissent expliquer au détail l'actualité du pays. Il réduit son apport, ainsi, à quelques « éclaircissements ». Sur le plan pictural, également, lorsqu'il choisit d'apparaître lui-même à l'écran, il ne cherche aucunement à se substituer aux sujets dont il filme les témoignages. Il se place, bien au contraire, à leur même 
niveau ; car son principal souci est de partager avec eux, à leurs côtés et non pas d'en haut, l'espace du cadre, d'où le trucage consistant à créer des plans composites par l'insertion intermittente d'un écran second - celui dans lequel il s'adresse aux spectateurs - au sein du plan principal ${ }^{9}$.

Ce procédé est notable pour deux raisons. D'une part, car il « [...] permet au spectateur d'identifier clairement les responsables, les porte-parole du message que l'on diffuse » (Beaucage, 2009 : 183). De l'autre, il se distingue en ce qu'il véhicule, par l'image, une hiérarchie éthique selon laquelle le commentaire reste subordonné à la parole des sujets interviewés. À tout moment - on le comprend alors - la préoccupation de Groulx sera de donner à chaque sujet « [...] toute la latitude pour faire valoir son point de vue » (ibid : 191). L'éloquence du dispositif choisi se trouve justement là, dans ce régime tout particulier de proximités qu'il autorise : partageant, par le procédé décrit, un seul et même espace, le commentaire du cinéaste et les témoignages des sujets se mélangent en effet pour produire des nouveaux sens ; mais, par la nette délimitation des bords du nouveau plan superposé, ils se tiennent en même temps à une distance prudente, ce qui empêche d'interrompre, de dénaturer la parole des intervenants.

(6) Recours au dialogisme : L'essai, en cinéma, contribue à une « [...] ouverture d'espaces de parole » (Terrier Herman, 2010 : 97). Or, ces espaces sont pour l'essentiel animés par la présence du réalisateur - personnage public lui-même -, qui dialogue avec les sujets interviewés ou avec le spectateur. Dans 24 heures ou plus, toutefois, Groulx empruntera une toute autre voie : à l'instar du Godard du Mépris, du Roi Lear ou même de Pierrot le fou (nous songeons à la très célèbre scène avec Samuel Fuller), Groulx choisira de transmettre ses pensées, si l'on veut, par personne interposée. Le geste, nous tenons à le souligner, est en harmonie avec les présupposés de l'essai, genre dont un des buts - Suzanne Liandrat-Guigues nous le rappelle - est d'exercer sa pensée en s'exerçant à la pensée d'autrui (2004 : 10).

Groulx fera figurer à l'écran, dans une longue séquence, le cinéaste Pierre Perrault, lequel s'entretient avec un groupe de militants pour les droits des amérindiens à l'occasion du lancement de la revue Recherches amérindiennes. La séquence, tournée en couleur, s'ouvre par l'entrée dans la pièce d'un de

9. À grands traits, ce trucage visuel consiste en l'incrustation d'un nouveau cadre, dont les bords resteront toujours visibles, au milieu du plan principal. Dans ces cadres seconds, qui apparaissent d'ailleurs de manière inopinée et intermittente et durent à peine quelques secondes, l'on verra indifféremment Gilles Groulx ou Jean-Marc Piotte lire des textes préparés à l'avance et s'adresser directement aux spectateurs. En termes de mise en scène, ces plans incrustés offrent en outre un contrepoint intéressant à la richesse de textures, couleurs et mouvements des images qu'ils se donnent pour but de commenter. Il s'agit, somme toute, de plans frontaux, fixes, dominés par une lumière bleuâtre, presque aseptisée, dans lesquels tout élément pouvant détourner l'attention du public de la parole des cinéastes est éliminé. 
ces militants amérindiens. Groulx, sitôt l'homme entré, effectue un arrêt sur image dont il se sert pour introduire un court texte qui parait trancher d'entrée la discussion : «Cet homme », lit-on alors, «n'a pas été entendu depuis 250 ans. Le moment est venu maintenant pour les Amérindiens de parler et pour les autres d'écouter ». La conversation qui s'en suit est quelque peu gênante et saccadée, incommode. Quitte à donner une image peu flatteuse de Perrault, Groulx réussit pourtant à faire passer, par l'intermédiaire de cette scène d'un dialogue entravé, un constat qui vient justifier son exhortation : il existe un gouffre irréductible, une incompréhension presque foncière séparant l'homme blanc bien-pensant et embourgeoisé du sort des opprimés.

(7) Prédilection pour le « mémorable » : À l'instar du Fond de l'air est rouge de Chris Marker - film avec lequel il dialogue à bien des égards -, 24 heures se veut, avant tout, un film-bilan, une sorte de compte-rendu traçant les événements survenus dans un intervalle de temps bien délimité - «les journées de novembre et décembre $1971 »-$ et dans une aire géographique spécifique «Montréal et ses environs ». Cette volonté de délimitation spatio-temporelle, du reste, est explicite depuis le tout début. Rappelons que, lors des premières séquences, la voix de Gilles Groulx annonçait déjà : « Nous avons tenté, avec une équipe réduite au minimum, d'enregistrer la réalité sociale au jour le jour, sachant qu'avec nos moyens il fallait compter sur la chance ». Dans ce même ordre d'idées, Beaucage nous rappelait, pour sa part, que 24 heures représente un effort pour dépeindre « [...] tout ce qui peut se dérouler en l'espace d'une journée ou d'une période temporelle plus prolongée, dans un Québec en pleine ébullition » (2009: 180).

En outre, ce désir de capturer un réel politique sur le vif n'est pas sans rappeler d'autres entreprises filmiques telles que La bataille du Chili de Patricio Guzmán ou Irak année zéro d'Abbas Fahdel, où il s'agissait également d'enregistrer au jour le jour, sans relâche, l'évolution d'une situation politico-sociale donnée dans son intégralité. Ce qui est à l'œuvre derrière de telles entreprises filmiques est, on le voit, un certain encyclopédisme, comme le note bien Véronique Terrier Hermann à propos des films d'Erik Bullot (2010 : 93). Or, cet encyclopédisme, dans le film-essai, ne cherche pas à « [...] exposer un savoir, [mais] suit le déroulement d'une pensée [...], joue plaisamment avec des savoirs » (ibid).

Dès lors, il apparaît clairement qu'il existe une tension, en cinéma, entre la volonté de donner une vision d'ensemble d'une réalité forcément complexe et la nécessité de suggérer cette même réalité par un agencement de fragments visuels épars, aussi cohérents qu'ils soient. Cette tension, Groulx et Piotte la remarquaient d'ailleurs dans leur texte d'introduction, dans lequel ils affir- 
maient : « Nous avons observé que les choses ne se produisent pas par hasard et qu'elles sont au contraire reliées par la réalité de l'ensemble qui, lui, est politique. Ça nous est apparu avec plus de clarté [...], au moment d'assembler les nombreuses scènes du film ».

$\mathrm{Au}$ fond, ce qui est en jeu ici est une articulation toute particulière - et propre au film-essai - entre la dispersion de la structure globale de l'œuvre et la force de chaque plan, de chaque événement, de chaque témoignage enregistrés. À ce propos, Groulx affirmait, toujours au début du film : «Il y a 56 sujets dans ce film, ce qui prouve encore une fois qu'il y a 56 façons de faire un film » ${ }^{10}$. Par cet aveu, le cinéaste québécois donnait à entendre qu'il ne s'agit aucunement pour lui d'engendrer un « retour du réel, une image captée de la réalité à l'état brut » (Terrier, 2010 : 93), mais plutôt de gérer « une mémoire sédimentée et mobile d'idées [...] [qu'il] relève, prélève, déstabilise » (Macé 2008 : 7). La conviction de Groulx, à ce sujet, ne laisse guère de place au doute : un film doit être, lui, aussi divers, volubile et fuyant que la réalité elle-même. Car c'est seulement dans cette circulation sans cesse de topiques «forme singulière de temporalisation du vrai », nous dit Marielle Macé - que le « long temps d'une culture commune » (Ibid) peut se manifester. Si chaque témoignage, si chaque sujet peuvent constituer l'objet d'un film différent, c'est qu'alors le réel, par ses plis et complexités inhérents, fonctionne déjà comme « une réserve existentielle, un réseau intime et partageable de citations » (Ibid : $8)$.

\section{Conclusion}

Loin du lyrisme folklorique auquel succombait parfois Perrault et à l'opposé du particularisme introspectif d'un Jutra, les films de Gilles Groulx rendent compte, dès ses premières réalisations, d'un désir vif pour capturer le réel dans ses contradictions et ses plis. Aussi déroutant qu'il puisse paraître à d'aucuns, son cinéma est dans tous les cas un univers indéniablement unique, intransférable, dans lequel se mêlent la quête formelle et la conscience croissante d'une lutte à mener avec urgence, ce qui le différencie à coup sûr du reste de ses collègues.

Ses œuvres sont - voici encore une autre caractéristique de l'essai - façonnées à son image. L'adjectif de «fébrile », que l'on pourrait accorder volontiers

10. David McIntosh, dans un article paru dans la revue canadienne Fuze, fait une énumération résumée de quelques-uns des sujets présentés dans le film. 24 heures ou plus, affirme-t-il, "[...] is an encyclopedic two-hour document of the lives of fifty-six different subjects, including farm workers, union leaders, newspaper editors, elected officials, housewives, hippies, garment workers, music students, bank robbers, food coop organizers, police attack dog trainers, zoo keepers, Cree land claims activists, a man who murdered his boss and his supportive wife [...]" (1996: 17). 
à ses films, est d'ailleurs employé par lui-même pour décrire sa personnalité : « Je vivais debout [...] Je suis trop fébrile », affirmait-il dans Trop c'est assez. Il n'est guère étonnant dès lors que Marielle Macé définisse l'essai comme un « [...] genre pressé, [...] toujours prêt à répondre aux sollicitations du monde » (2008 : 6). L'image, si l'on considère l'importance, pour l'essai, de la notoriété personnelle de l'auteur, est parlante. Rappelons qu'à propos de Montaigne, Jean Starobinski disait déjà, pour évoquer les évolutions de sa pensée : ses mains «[...] étaient toujours en mouvement» $(2003: 171)$, son esprit et son corps «ne [restaient] pas aisément en repos » (Ibid).

Et c'est notamment sur cette fébrilité, sur cet empressement foncier, que 24 heures ou plus repose. Car, pour Groulx, il s'agit avant tout de faire revivre, à l'aide d'éléments disparates qu'il agence avec astuce, l'effervescence politique d'un Québec secoué par les événements récents de la Révolution tranquille. Son mérite, cependant, consiste à en donner une vision personnelle, authentique, inédite, «à partir de matériaux sonores et visuels dont la structuration ou combinaison non seulement laisse visibles les traces d'un processus de pensée, mais les incorpore à la texture même du film et joue sur leurs tensions » (Moure, 2004 : 36).

En cela, 24 heures ou plus répond positivement à la question que Jacques Aumont se posait sur les possibilités pour un film de constituer un acte de théorie. Rappelons à cet égard que pour Aumont le terme «théorie » recouvrait trois noyaux : la spéculation, la systématicité et la puissance explicative, tous les trois présents, bien qu'à des degrés distincts, dans le film analysé. Car 24 heures, tout d'abord, prétend à « expliquer, au moins potentiellement, un phénomène » (2007:200), ce qu'il fait d'ailleurs par l'intermédiaire d'un dispositif cohérent et systématique ; mais le film possède aussi - et voilà son trait le plus intéressant - un aspect spéculatif au sens où il enquête sur « les conditions d'une expérience qui a rapport avec l'expérience filmique » (Aumont, 2007 : 202) et vis-à-vis de laquelle il est en mesure d'apporter de réponses. Cette expérience est, en l'occurrence, celle de l'éveil politique et de l'émancipation du Québec au sortir de la Révolution tranquille.

Il est en tout cas étonnant de constater que Groulx se servait de ce même mot lorsqu'il cherchait à expliquer, dans Trop c'est assez, le métier du monteur, qu'il a d'ailleurs toujours revendiqué : « un monteur », disait-il, « c'est plus ou moins un spéculateur. C'est un gars qui joue avec la matière puis qui aime ça » (Brouillette, 1995). Par son recours aux métaphores du jeu, de l'amour et de la matière, Groulx pointait, peut-être sans le savoir, à l'une des principales caractéristiques de l'essai ; à savoir, sa capacité à façonner, de manière méditative, et avec les moyens qui lui sont propres, une matière extérieure, objectuelle, un 
réel déjà découpé (et non une réalité à l'état brut) qui impose en quelque sorte sa propre logique. Veronique Terrier Hermann éclaircit la question, à l'aide de formules davantage éloquentes et succinctes : «L'essai », affirme-t-elle, « fonctionne comme réflexion sur le monde à partir d'une remise en forme du réel » (2010:89-90); « pour [lui] faire le choix d'un sujet c'est faire le choix d'une forme esthétique » (Ibid : 92).

Le champ des belles-lettres, quant à lui, abonde d'exemples de cette conception dite objectuelle. Contentons-nous de n'en citer que deux. Adorno, sur le sillage de Benjamin et Simmel, apparentait ainsi l'essai - le même mot revient encore une fois - à une «[...] spéculation sur des objets spécifiques, déjà formés d'avance dans la culture » (2003 : 50). Lukács, pour sa part, affirmait : « [L]essai parle toujours de quelque chose qui possède déjà une forme, ou au mieux de quelque chose qui a déjà été $[\ldots]$ » (1972 : 104). Et faudrait-il citer encore Jean Starobinski, lequel écrivait, à propos des Essais de Montaigne : «Le champ d'expérience, pour [lui], est d'abord le monde qui lui résiste : ce sont les objets que le monde offre à sa prise [...] $\gg(2003: 171)$. Groulx, pour sa part, commente :

[...] le documentaire est particulièrement très difficile, infiniment plus compliqué que le long-métrage [de fiction]. On ne dispose pas, comme dans le film dramatique de long-métrage, de l'action de boomerang que crée l'existence des personnages. On fait à partir d'objets, et même si ce sont des gens qui marchent dans la rue. Ça reste quand même, du point de vue de l'observant, des objets inanimés, qui portent à toute autre interprétation. Et on doit prendre parti. (Chouinard, 2002 Entretien).

Les convictions de Groulx à cet égard ne laissent guère de place au doute : par le biais de la monstration d'un réel objectuel, et de la réorganisation, de l'agencement de ses composants, le cinéma mettrait le spectateur devant un choix à faire et exigerait de lui, sinon un passage à l'acte, au moins une prise de position claire. S'il en était autrement, la question qu'il pourrait poser ne serait pas valable, car biaisée. En effet, c'est seulement par le truchement du réel que le cinéma peut parvenir, selon Groulx, à toucher, à affecter, de quelque manière qu'il soit, le monde. Une approche non objectuelle équivaudrait, pour le réalisateur, à un faussement de la réalité et, par conséquent, à un piège, voire même à de l'aliénation. C'est en tout cas, selon lui, le langage documentaire qui le veut ainsi.

Récapitulons. Au fil de ces pages nous avons essayé simplement de montrer de quelle manière 24 heures ou plus répond aux caractéristiques de ce que la littérature connait sous le nom de « film-essai ». Or, ce qui nous intéressait de souligner, spécifiquement - d'où le titre de l'article -, c'était qu'il existe chez Groulx non seulement - cela va de soi - une pratique essayiste, mais aussi, et 
plus fondamentalement, une théorie embryonnaire des capacités du cinéma à véhiculer des réalités complexes, de constituer, enfin, une pensée, d'ouvrir sur un processus. Donnons-lui la parole, pour conclure :

Le film, même en préparation, est beaucoup plus qu'une 'rédaction'; il tient d'avantage de l'intuition de l'inventeur qui s'accroche à quelques signes perçus et qui commence à grands traits à faire le croquis de son plan. Le film est lumière et son; le film est idée et intentions; le film est perception et exposition; le film est invention et création dans une collaboration, depuis le commencement jusqu'à la fin. (Groulx, $1986: 3$ ).

\section{Bibliographie}

Adorno, T. (2003). L'essai comme forme. Approches de l'essai. Anthologie. Québec : Éditions Nota Bene.

Astruc, A. (1992). Du stylo à la caméra ... et de la caméra au stylo : Écrits (1942-1948). Paris : L'Archipel.

Aumont, J. (2007). Un film peut-il être un acte de théorie ?. Cinémas, 172(3) : 193-211.

Blümlinger, C. (2004). Lire entre les images. In S. Liandrat-Guigues \& M. Gagnebin (dir.), L'essai et le cinéma. Seyssel : Éditions Champ Vallon.

Beaucage, P. (2009). Gilles Groulx, le cinéaste résistant. Montréal : Lux.

Comeau, R. (1995). La Révolution tranquille, une invention ?. Cap-aux-Diamants, (41), 52-56.

Daudelin, R. (1967). Vingt ans de cinéma au Canada français. Québec : Éditions du Ministère des Affaires Culturelles.

Dulac, G. (1994). Écrits sur le cinéma (1919-1937). Paris : Paris expérimental.

Froger, M. (2009). Le cinéma à l'épreuve de la communauté. Le cinéma francophone de l'Office national du film. 1960-1985. Montréal : Les presses de l'Université de Montréal.

Gagnebin, M. (2004). L'inconscient à l'essai. In S. Liandrat-Guigues \& M. Gagnebin (dir.), L'essai et le cinéma. Seyssel : Éditions Champ Vallon.

Groulx, G. (1986). Propos sur la scénarisation. Laval, Québec : Éditions de la Cinémathèque Québecoise.

Harcourt, P. (1992). Naissance du direct : 1960-1970. In S. Garel \& A. Pâquet (dir.), Les cinémas du Canada. Paris : Éditions du Centre Georges Pompidou. 
Jeancolas, J.-P. (1992). La révolution par l'album : 1970-1980. In S. Garel \& A. Pâquet (dir.), Les cinémas du Canada. Paris : Éditions du Centre Georges Pompidou.

Langlet, I. (2015). L'abeille et la balance. Paris : Classiques Garnier.

Liandrat-Guigues, S. (2004). Un art de l'équilibre. In S. Liandrat-Guigues \& M. Gagnebin (dir.), L'essai et le cinéma. Seyssel : Éditions Champ Vallon.

Lever, Y. (1995). Histoire générale du cinéma au Québec. Montréal : Boréal.

Lukács, G. (1972). Nature et forme de l'essai. Études littéraires, 4(1) : 91-114.

Macé, M. (2008). L'essai littéraire, devant le temps. Cahiers de narratologie, 14 : 1-10. En ligne : consulté le 5 octobre 2016.

McIntosh, D. (1996). 360 degrees of separation : notes on facts and fictions of self and nation in the recurring Quebec referendum. Fuse Magazine, 19(2) : 14-20. En ligne : http://openresesarch.ocadu.ca/id/eprint/1903/

Moure, J. (2004). Essai de définition de l'essai au cinéma. In S. LiandratGuigues \& M. Gagnebin (dir.), L'essai et le cinéma. Seyssel : Éditions Champ Vallon.

Noguez, D. (1987). Le cinéma, autrement. Paris : Les Éditions du Cerf.

Pâquet, A. (1986). Le cinéma québécois se laisse aller à un glissement progressif de désir commercial. CinémAction, (40).

Patenaude, M. (1964). Entretien avec Gilles Groulx. Objectif, 64(29-30) : 3-14.

Starobinski, J. (2003). Peut-on définir l'essai ?. In Approches de l'essai. Anthologie. Québec : Éditions Nota Bene.

Terrier Hermann, V. (2010). Cinéma et art contemporain, nouvelles approches de l'essai. Marges, $10: 86-100$.

Zéau, C. (2006). L'Office National du Film et le cinéma canadien (1939-2003) : éloge de la frugalité. Bruxelles : Peter Lang.

\section{Filmographie}

Entretien en six temps avec Gilles Groulx (2002) de Denis Chouinard.

Voir Gilles Groulx (2002) de Denis Chouinard.

Trop c'est assez (1995) de Richard Brouillette.

24 heures ou plus $(1973,1977)$ de Groulx, Gilles. 